\title{
Estrus response and follicular development in boer does synchronized with flugestone acetate and PGF2 $\alpha$ or their combination with eCG or FSH
}

\begin{abstract}
The effects of different estrus synchronization techniques on follicular development and estrus response were studied in 81 nulliparous Boer does. The does were divided into nine groups. Eight of the nine groups were synchronized with prostaglandin F2-alpha (PGF2 $)$ or flugestone acetate (FGA) or their combinations, and the ninth group was a control group. In addition to the above combinations, four of the eight synchronized groups were given $5 \mathrm{mg}$ follicle-stimulating hormone (FSH) and the remaining four groups were administered $300 \mathrm{IU}$ equine chorionic gonadotrophin (eCG). Posttreatment follicular development was monitored until ovulation occurred using a real-time B-mode ultrasound scanner (Aloka, 500 SSD, Japan), with a 7.5-MHz transrectal linear probe. All the does from the synchronized groups that were given eCG exhibited oestrus while only $88.9 \%$ of the does synchronized with FSH showed estrus. The estrus response was observed to be the least among the does synchronized with PGF2Ŭ + FSH (33.3\%) combination followed closely by the FGA + FSH (42.9\%) combinations. It was observed that the combinations of FGA + PGF2Ŭ + FSH resulted in increased percentage of estrus response, duration of estrus, and ovulation. The number of follicles was higher (P\&lQ0.05) in FSH-synchronized groups than the eCG-synchronized groups. It was concluded that the best estrus synchronization protocol in goats is the FGA + eCG with or without PGF2Ŭ. However, the PGF2Ŭ + FGA + FSH method of estrus synchronization is the most promising combination for further development as a better alternative to estrus synchronization with eCG in does.
\end{abstract}

Keyword: Boer goat; Flugestone acetate; Gonadotrophins; Estrus synchronization; PGF2ŭ, Ultrasonography 